\title{
LEP2: PRESENT AND FUTURE PERFORMANCE AND LIMITATIONS
}

\author{
S. Myers (for the LEP2 team), CERN, Geneva, Switzerland
}

\begin{abstract}
The LEP collider was operated during 1996 for the first time at beam energies beyond the $\mathrm{W}$ pair threshold. In the first period, lasting about six weeks, operation was possible at $80.5 \mathrm{GeV}$ per beam by powering all of the 144 superconducting cavities (sc) as well as the original 120 room temperature cavities. After the summer shutdown, when an additional $32 \mathrm{sc}$ cavities were installed, operation for physics was performed at $86 \mathrm{GeV}$ per beam for about four weeks. The integrated luminosity at high energy, delivered to the detectors, was $25 \mathrm{pb}^{-1}$ in the two fairly short running periods. The maximum integrated luminosity over a 24 hour period exceeded $1.1 \mathrm{pb}^{-1}$. The present performance and limitations of the machine are reviewed as well as some of the crucial technical systems.
\end{abstract}

\section{INTRODUCTION}

The CERN Large Electron Positron (LEP) collider is a $26.6 \mathrm{~km}$ circumference $\mathrm{e}^{+} \mathrm{e}^{-}$storage ring which has, until the end of 1995, operated with 4 and 8 bunches per beam in an energy range of 20 to $50 \mathrm{GeV}$ (see previous conference reports, [1],[2])

The energy upgrade (LEP2), foreseen to allow study of $\mathrm{W}$ pairs at energies around $90 \mathrm{GeV}$ per beam, involves a total of 272 new sc cavities with gradients of $6 \mathrm{MV} / \mathrm{m}$. In addition to the sc cavities the following technical modifications were necessary for the upgrade.

- Civil engineering for klystron galleries. Two new klystron galleries were excavated near points 4 and 8 .

- 4 new $12 \mathrm{~kW}$ (at $4.5 \mathrm{~K}$ ) cryogenics plants were installed in the four regions which house sc cavities.

- The RF straight-sections were rearranged in all four interaction regions to allow the installation of the sc cavities which had different lengths to the existing copper cavities.

- The sc low $\beta$ insertion quadrupoles were upgraded for the higher energy, as were nearly all of the power converters

\section{PERFORMANCE AND HIGHLIGHTS OF 1996}

Due to the long shutdown periods imposed by the installation of the sc cavities, the available time for physics in 1996 was limited to about 10 weeks. The initial physics operation was foreseen with an optics configuration with $108^{0}$ phase advance per cell in the horizontal plane, designed to produce higher specific luminosity due to the inherent smaller horizontal emittance. It was soon discovered that the usable dynamic aperture was significantly lower than

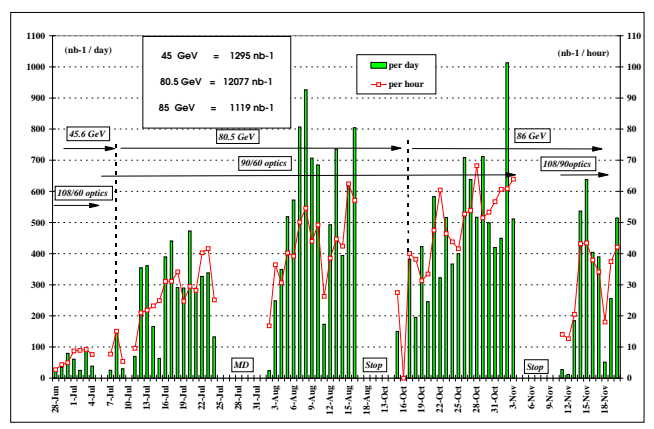

Figure 1: Measured Daily and Hourly Luminosity for 1996

computer simulations had predicted. For this reason, after nearly three weeks of attempted running the sextupoles were recabled and the older, well tried $90^{\circ}(\mathrm{H})$ and $60^{\circ}(\mathrm{V})$ optics was reinstalled in the machine and re-commissioned. Physics conditions were soon re-established and the collider was operated initially at $80.5 \mathrm{GeV}$ per beam and, after an additional installation of $32 \mathrm{sc}$ cavities in the summer, at 86GeV. The performance for 1996 is shown in Fig.1.

Although the maximum operational beam energy for physics production was $86 \mathrm{GeV}$, a record single beam energy of $88 \mathrm{GeV}$ was reached by operating the RF at the allout limit. In this mode of operation, the average gradient of the $176 \mathrm{sc}$ cavities was the design value of $6 \mathrm{MV} / \mathrm{m}$ and a spurious trip of a single module would inevitably cause total beam loss. In "operational mode" the total voltage available is such that, in the event of simultaneous tripping of 2 klystron (feeding 16 cavities) there remains sufficient $\mathrm{RF}$ voltage for a longitudinal quantum lifetime of around 15 hours.

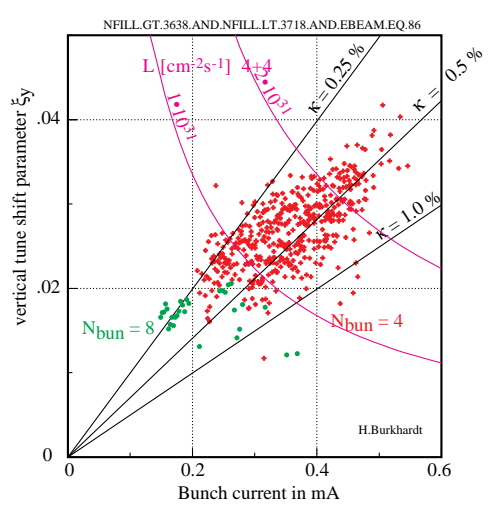

Figure 2: Measured $\xi_{y}$ as a Function of Bunch Current

After initial conditioning and commissioning, the RF system behaved very reliably at its design gradient and with a total beam current of $4 \mathrm{~mA}$. The current was limited by 
the "electro-acoustic" instability [3] in the cavities, and for normal operation the cavity conditions were optimized for this intensity value. Although the total current was limited, a record peak luminosity of $3.4 \times 10^{31} \mathrm{~cm}^{-2} \mathrm{~s}^{-1}$ was reached due to the very small emittance ratio of $0.25 \%$ achieved (refer to Fig.2). The maximum integrated daily luminosity reached $1.1 \mathrm{pb}^{-1}$ (see Fig.1) which bodes well for 1997. For 1997 a new feedback system (which was tested on a limited number of cavities in 1996) will be commissioned to combat the cavity instability [3]. During machine studies, single bunch currents of more than $600 \mu \mathrm{A}$ were successfully accumulated and ramped to high energy.

The $4 \times 12 \mathrm{~kW}$ cryogenic plants were operated without serious problems and with high reliability throughout the year [4].

On the beam dynamics front the vertical beam-beam tune shift $\left(\xi_{y}\right)$, as measured from the average of the luminosities in the four detectors, reached values $\geq .04$ (see Fig.2) without any signs of saturation.

In LEP the precision measurement of the beam energy is of crucial importance for the precision of the physics. For LEP1 the beam energy was measured around the $\mathrm{Z}^{0}$ equivalent energy by transverse resonant depolarization. For LEP2 it is likely that the polarization level at high energies will be too small to be measured. Hence the beam energy at $\mathrm{W} \pm$ must be estimated by extrapolation from resonant depolarization at lower energies [5]. The extrapolation will be performed by the use of NMR monitors and the LEP "flux loop". The accuracy of the extrapolation depends on the energy range over which the calibrations can be performed. Precision measurements have been systematically performed at 43 and $45 \mathrm{GeV}$ for the $\mathrm{Z}^{0}$ data. In addition, in 1996, a successful calibration was performed for the first time at $50 \mathrm{GeV}$. An attempt was also made to extend the calibration to $55 \mathrm{GeV}$ where, although polarization levels of $2 \%$ were measures no accurate calibration could be performed.

\section{PERFORMANCE LIMITATIONS}

The two most important parameters for consideration of the performance of LEP2 are:

- the maximum beam energy, and

- the integrated luminosity

\subsection{Maximum Energy}

The over-riding limitation to the beam energy comes from the available RF voltage. The available RF is simply the product of the active length of the cavities and the average gradient per meter. The former limit is set by funding and the latter by technological constraints.

The energy spread of the RF bucket is approximately given by

$$
\left(\frac{\Delta E}{E}\right)_{b k} \simeq Q_{h} \sqrt{\frac{e V_{R F}}{h E_{b}} f\left(\phi_{s}\right)}
$$

Hence, given the RF installation, an optics with stronger focussing provides relatively larger energy spreads and therefore allow a larger fraction of the RF voltage to be used to compensate synchrotron radiation, thereby permitting higher energies. In LEP2 the difference between the $90^{\circ}$ and the $108^{\circ}$ horizontal phase advance per cell is approximately $0.8 \mathrm{GeV}$ at $90 \mathrm{GeV}$ beam energy.

In the transverse plane there is an additional advantage to stronger focusing optics coming from the horizontal emittance.

$$
\epsilon_{x} \propto \frac{\gamma^{2}}{Q_{h}^{3} J_{x}}
$$

Clearly, for a dynamic or physical aperture which is independent of energy, then with the horizontal emittance growing with $\gamma^{2}$, at a high enough energy the aperture will be insufficient. This situation can be alleviated by operating with the "high tune optics". The alternative is to reduce the horizontal emittance by increasing the damping partition numbers $\left(J_{x}\right)$ which unfortunately increases the energy spread of the bunches and thereby reduces slightly the maximum energy.

In general substantial gains in beam energy require either more RF cavities or higher gradients. The constraints on the LEP optics is treated in detail elsewhere in this conference [6].

\subsubsection{Dynamic Aperture}

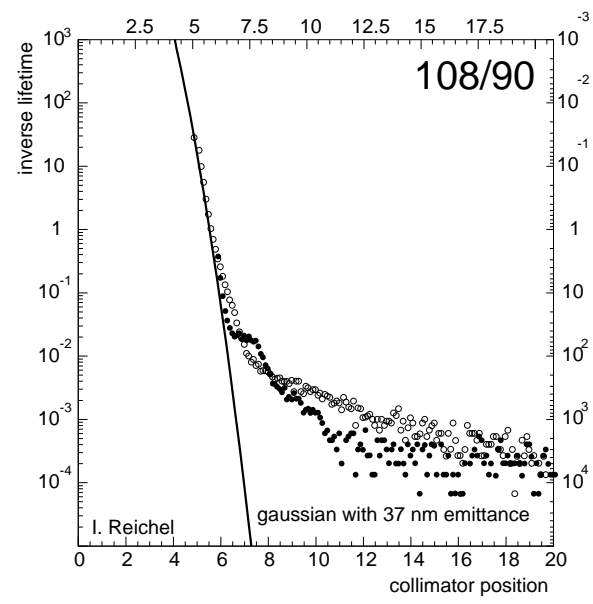

Figure 3: Measurement of Tail Distribution with $108^{0} / 90^{0}$ Optics

As stated previously, the usable dynamic apertures with the $108^{\circ} / 60^{\circ}$ and later in the year with the $108^{\circ} / 90^{\circ}$ optics were measured to be less than predicted by simulations. The dynamic aperture is measured in LEP in two independent ways, firstly by the "emittance inflation" technique where the damping partition numbers are varied so as to increase the horizontal emittance by a large factor. When the emittance becomes large enough to reduce the lifetime this defines the dynamic or physical aperture. The inflated emittance is measured by a carefully calibrated synchrotron light monitor and verified by measuring the tails of the distribution using loss monitors [7]. Figure 3 shows the 
results of tails scans on the $108^{\circ} / 90^{\circ}$ optics. It is clear that non-Gaussian tails exist at large amplitudes. Similar measurements with the $90^{\circ} / 60^{\circ}$ optics showed that the development of tails ocurred at larger amplitudes. In practice these non-Gaussian tails can produce serious problems with background in the detectors before there is a serious reduction in the lifetime.

The second technique, the "kicker technique", involves deflecting the beam horizontally by an ever increasing amount until a sizeable fraction of the beam is lost thereby indicating that the aperture limitation had been reached. In general, both methods gave similar results, however the results of the kick method can be easier to interpret and with suitable diagnostics has indicated the presence of nonlinear resonances at large amplitudes. This behaviour will be studied during 1997 (see [8] for a fuller description of these measurements and analyses).

\subsection{Integrated Luminosity}

The yearly integrated luminosity may be conveniently approximated by

$$
\int L d t=\eta L_{\text {peak }} t_{\text {year }}
$$

where $\eta$ is the "overall efficiency factor" and $L_{\text {peak }}$ is the all-out maximum luminosity recorded in physics during the year.

The luminosity $(L)$ is directly related to the vertical beam-beam tune shift $\left(\xi_{y}\right)$ i.e.

$$
L=\frac{\gamma}{2 r_{e} e} \frac{k_{b} i_{b} \xi_{y}}{\beta_{y}^{*}}
$$

where $\gamma$ is the relative energy, $k_{b}$ the number of bunches per beam, and $i_{b}$ the bunch current.

\subsubsection{The Overall Efficiency $(\eta)$}

The overall efficiency parameter $(\eta)$ is derived by using historical data and equation (3). Consequently this parameter includes factors such as:

- The loss in integrated luminosity since all runs do not necessarily reach the record luminosity $\left(L_{\text {peak }}\right)$.

- The fall in luminosity during the course of the run due to the intensity lifetime

- The technical efficiency (down-time) of all components including the chain of injectors, defined as the ratio of actual time spent in physics data taking to the time scheduled.

The Intensity Lifetime In LEP, the intensity lifetime $(\tau)$ is predominated by the particle losses resulting from the collisions of the electrons and positrons in the 4 collision points.

$$
\frac{1}{\tau}=\frac{d I_{b}}{I_{b} d t}=\frac{\sigma_{b} L n_{\text {collisions }}}{I_{b}} \propto \frac{\sigma_{b} \xi_{y} \gamma n_{\text {collisions }}}{\beta_{y}^{*}}
$$

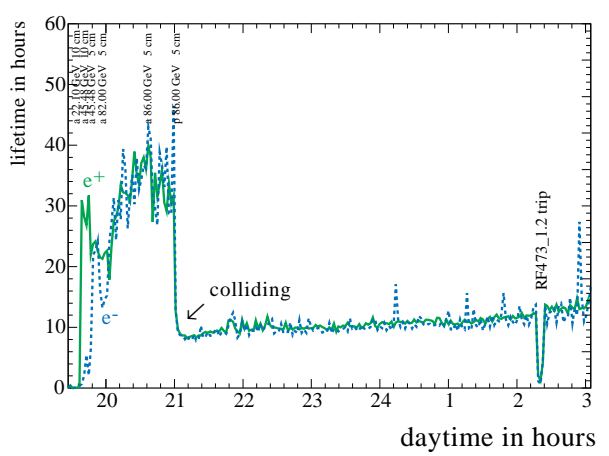

Figure 4: Lifetime Measurements during an $86 \mathrm{GeV}$ Fill

where $I_{\mathrm{b}}$ is the total beam current, and $\sigma_{b}$ is the crosssection. With the typical LEP parameters $\left(n_{\text {collisions }}=\right.$ $4, \sigma_{b}=.23$ barns (measured), $\beta_{y}^{*}=5 \mathrm{~cm}$ ) the intensity lifetime is approximately

$$
\tau(\text { hours }) \approx \frac{1}{\xi_{y}} \frac{45}{E_{G e V}}
$$

Figure 4 shows measurements [9] of the intensity lifetime during a typical physics run, involving accumulation at $22 \mathrm{GeV}$, acceleration to $86 \mathrm{GeV}$ and colliding. The lifetime clearly drops by nearly a factor of 4 at the moment of going into collision and gradually increases during the duration of the physics run due to the reduction in $\xi_{y}$.

From equations (5) and (6) the intensity lifetime will decrease proportionately with energy. Consequently the durations of the runs at $\mathrm{W} \pm$ energies are shortened so as to optimize the integrated luminosity.

\subsubsection{Beam-beam Contribution}

From equation (4), it is clear that, when $\xi_{y}$ remains constant during the physics run (i.e. at the beam-beam limit) then the luminosity lifetime is equal to the intensity lifetime. Alternatively when the $\xi_{y}$ is linearly dependent on the bunch current (not beam-beam limited) then the luminosity lifetime is equal to the lifetime of $i_{b}^{2}$. Thus in order to maximize the integrated luminosity it is essential to remain beam-beam limited to the end of the physics run when the intensity has significantly dropped. In LEP1, this was accomplished [9] without difficulty due to the smaller beam sizes associated with the lower energies (see equation 2). The vertical beam-beam tune shift may be written

$$
\xi_{y}=\xi_{x} \sqrt{\frac{\beta_{y}^{*}}{\beta_{x}^{*} k}} \propto \frac{i_{b} Q_{h}^{3} J_{x}}{\gamma^{3}} \sqrt{\frac{\beta_{y}^{*}}{\beta_{x}^{*} k}}
$$

where $k$ is the emittance ratio $\epsilon_{y} / \epsilon_{x}$. For LEP2 where the energy is more than doubled, it will be necessary to gain about a factor of 8 in the combination of the free parameters in equation (7) if a similar beam-beam situation similar to $\mathrm{Z}^{0}$ is to be achieved.

\subsubsection{Beam Intensity}

In LEP2, there are effects which limit the total beam current and others which limit the current per bunch and the 
number of bunches. For LEP2 the limitations to the total beam current are given below starting with the highest limits and progressing to the lowest, most urgent limitations.

1. The power which can be transmitted through the main couplers. The couplers have been tested to $300 \mathrm{~kW}$ per cavity which would correspond to $2.4 \mathrm{MW}$ equivalent from the klystrons, to be compared with their maximum power of 1.3 MW. This is no longer a limitation to beam intensity.

2. The total available klystron power which is available to compensate for the synchrotron power. (e.g. 30 klystrons giving $30 \mathrm{MW}$ would allow a total beam current of $16 \mathrm{~mA}$ at $90 \mathrm{GeV}$ ).

3. The electro-acoustic instability.

4. The current (and Energy) dependent cryogenic losses (see below)

Beam Current Provoked Cryogenic Power Losses Towards the end of 1996 measurements of the cryogenic losses with varying beam conditions indicated unexpected losses which were apparently dependent on the total bunch intensity and the beam energy [10].

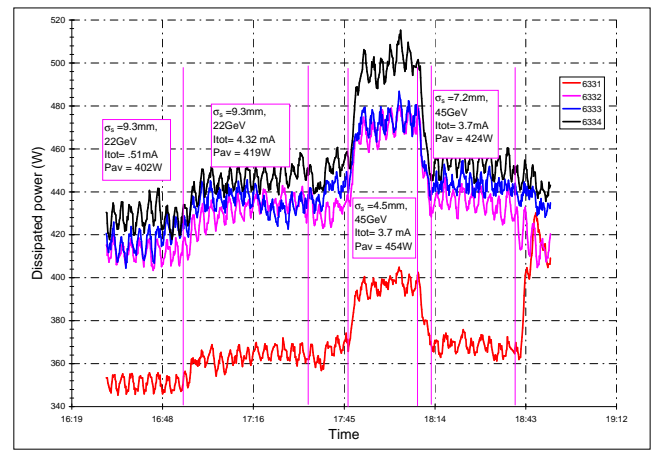

Figure 5: Measured Changes in Cryogenic Loads with Changing Beam Conditions

Fig. (5) shows the relative power changes during beam accumulation at $22 \mathrm{GeV}$, followed by ramping to $45 \mathrm{GeV}$ where the bunch length was changed by excitation of wiggler magnets. The variation of the cryogenic power was also measured during the course of a physics run at $86 \mathrm{GeV}$ and is plotted as a function of beam current squared in Fig.(6). Superimposed on this plot are the measured points from Fig (5). There is a clear increase in the slope of about a factor of 4 between 22 and $86 \mathrm{GeV}$. Similar results were obtained from measurements of the temperature rise of warm bellows between the sc modules. These results are however not irrefutable since the measurements were taken on different modules at different locations. However if the measurements made on the modules at $86 \mathrm{GeV}$ in Fig. (6) can be applied to all modules installed in a given region in LEP, then the total beam intensity may be limited to around $8 \mathrm{~mA}$ with the presently installed and finely tuned cryogenics plant. A complete measurement campaign of this effect is planned as soon as LEP becomes operational in 1997.

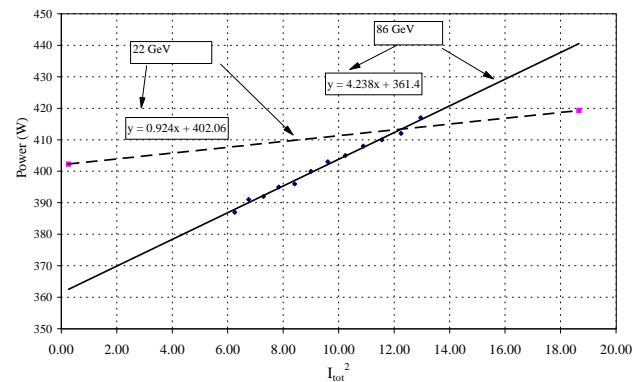

Figure 6: Crgryogenic Power Measurements as Function of $\mathrm{I}_{b}^{2}$ for 22 and $86 \mathrm{GeV}$.

Transverse Mode Coupling Instability This instability limits the current per bunch which can be accumulated at injection energy.

The approximate threshold for the instability is given by

$$
i_{t h}=\frac{2 \pi Q_{s} E f_{r e v}}{e \sum \beta_{i} k_{\perp i}\left(\sigma_{s}\right)}
$$

where $Q_{s}$ is the synchrotron tune, $E$ the beam energy, and $\beta_{i}$ the betatron amplitude function at the location of the transverse loss factor $k_{\perp i}$ which decreases with increasing bunch length $\left(\sigma_{s}\right)$. In order to maximize the current per bunch for LEP2 operation, several schemes have been tested and reported in previous conferences [1],[11]

Number of Bunches LEP has been operated in 1995 and throughout 1996 with the new bunch train scheme allowing a maximum of 4 trains of 4 bunches per train in each beam. A detailed report on this scheme is to be presented elsewhere in this conference ([12]). It is foreseen to operate LEP during 1997 with 4 trains of 2 bunches per beam.

\section{PERFORMANCE ESTIMATES (1997-1999)}

Table 1: Performance Estimates

\begin{tabular}{|l|c|c|c|c|}
\hline Parameter & $\mathbf{4 5} \mathbf{~ G e V}$ & $\mathbf{9 2 ~ G e V}$ & $\mathbf{9 6} \mathbf{~ G e V}$ & $\mathbf{9 6} \mathbf{~ G e V}$ \\
\hline & $\mathbf{1 9 9 4}$ & $\mathbf{1 9 9 7}$ & $\mathbf{1 9 9 8}$ & $\mathbf{1 9 9 9}$ \\
\hline No. of sc cavities & & 240 & 272 & 272 \\
\hline 'Operational' RF Voltage(MV) & & 2475 & 2722 & 2722 \\
\hline Overall Efficiency & 0.220 & 0.165 & 0.165 & 0.165 \\
\hline i $_{\text {b (bunch mA) }}$ & 0.32 & 0.5 & 0.65 & 0.75 \\
\hline Beam-beam tune shift & 0.038 & 0.0417 & 0.045 & 0.05 \\
\hline L peak (l0e31) & 2 & 6.86 & 10 & 13.3 \\
\hline Days of Physics & 148 & 108 & 120 & 120 \\
\hline Yearly Luminosity (pb-1) & 65.0 & 122.2 & 198.0 & 263.3 \\
\hline Luminosity per day (pb-1) & 0.439 & 1.132 & 1.650 & 2.195 \\
\hline
\end{tabular}

The performance estimates for operation over the next three years (see Tab.1) are based on equation (3) with extrapolation from the year 1994 (the last full year of uninterrupted operation) and the following assumptions.

- the overall efficiency parameter $\eta$ will be only $75 \%$ of the value attained (22\%) at lower energy in 1994. This reduction is to take account of the possible increase in down time due to the large number of sc cavities and the reduction in integrated luminosity coming from 
the increased difficulty in maintaining the beam-beam limit right to the end of the run as was done systematically at $45 \mathrm{GeV}$.

- The peak luminosity is estimated from 8 bunches per beam with a bunch current of .5mA. Both these parameters have bee attained in the past however it remains to be seen if the inherent total beam current of $8 \mathrm{~mA}$ will exceed the limit imposed by the cryogenics system.

- The total numbers of days operation is already scheduled definitively for 1997 and tentatively for 1998 .

From this table it can be seen that the peak luminosity is estimated to reach beyond $10^{32} \mathrm{~cm}^{-2} \mathrm{~s}^{-1}$ and the total integrated luminosity over the three year period to exceed $500 \mathrm{pb}^{-1}$.

\section{MAXIMUM ENERGY WITH PRESENT RF INSTALLATION}

Studies have begun recently to examine the possibility of extending the present beam energy range of LEP2 to around $100 \mathrm{GeV}$ with the presently installed RF system. This increase in beam energy would necessitate an average gradient in the $272 \mathrm{sc}$ cavities of $7 \mathrm{MV} / \mathrm{m}$. The problems already encountered and the solutions being pursued are given below.

- Cryogenics Power. From the $12 \mathrm{~kW}$ cryogenics power installed at each interaction point there remains about $6.8 \mathrm{~kW}$ for the RF dynamic load. At the higher gradients of $7 \mathrm{MV} / \mathrm{m}$ and with the resulting reduction in $\mathrm{Q}$ value, the calculated required power is at least $10 \mathrm{~kW}$. Consequently the cryoplants must be upgraded before any significant increase in gradient is possible. The proposed solution is to plan an early installation of the required LHC cryogenics system which could be used by LEP2 from Spring 1999.

- Electron emission in the cavities. The present gradient of $6 \mathrm{MV} / \mathrm{m}$ is for the most part limited by electron emission in the cavities with resulting high radiation. The proposal is to increase the threshold by Helium processing. This technique has been successfully tested on a number of occasions to reduce the electron emission at values below $6 \mathrm{MV} / \mathrm{m}$ in "sick" cavities. However the procedure is inherently dangerous for the main couplers due to the significant reduction in vacuum pressure needed for the processing. Experimental tests are under way to try to develop a safe He processing technique.

- Spread in cavity gradients. When an RF module (8 cavities fed by one klystron) is operating at an average gradient per cavity of $6 \mathrm{MV} / \mathrm{m}$, the spread in the gradients is typically more than $\pm 1 \mathrm{MV} / \mathrm{m}$. Consequently some of the cavities in a module are already being operated at $7 \mathrm{MV} / \mathrm{m}$. It is clear that an average of $7 \mathrm{MV} / \mathrm{m}$ will be more easily obtained if the spread of gradients in the modules is significantly reduced. This technical problem has been studied and one possible solution is to install waveguide transformers to allow individual control of the cavity gradients.

\section{CONCLUSIONS}

LEP2 has been sucessfully operated for a reduced running period during 1996. Physics data taking was performed at $80.5 \mathrm{GeV} /$ beam in the early part of the year and $86 \mathrm{GeV}$ later in the year. A total integrated luminosity of $25 \mathrm{pb}^{-1}$ was delivered to each of the four experiments and many technical problems were resolved with the newly installed very large RF system. The down-time of this system decreased dramatically during the course of the year as the steep learning curve was climbed.

Operation is planned for around 120 days in 1997 at $92 \mathrm{GeV} /$ beam allowed by the $240 \mathrm{sc}$ cavities installed over the past few years. There is every hope for an integrated luminosity of nearly $100 \mathrm{pb}^{-1}$ in the search for new physics and for the precision study of the properties of the $\mathrm{W} \pm$ particles.

The possibility of further extension of the LEP2 energy range is under active investigation.

\section{REFERENCES}

[1] S. Myers; "LEP Status and Plans", Proc. of the US particle Accelerator Conference and the 16th Int. Conf. on High Energy Accelerators, Dallas USA. (1995).

[2] A. Hofmann; "Performance Limitations in LEP", Proc. of the Fourth European Particle Accelerator Conference, held in London from 27 June until 1 July, 1994. (pp 73- 77), (1994)

[3] D. Boussard; "Performance of the LEP2 SRF System", invited contribution to this conference.

[4] P. Lebrun; "Cryogenics for Particle Accelerators; Present State of the Art and Future Trends", invited contribution to this conference.

[5] M. Böge et al., "Transverse Polarization beyond the Z Energy in LEP", contribution to this conference.

[6] D. Brandt et al., "Low Emittance lattices for LEP", contribution to this conference.

[7] I. Reichel et al.; "Observation and Simulation of Beam Tails in LEP", contribution to this conference

[8] J.M. Jowett; "Non-Linear resonances: Predictions, Effects and Measurements", Proc. 7th LEP Performance Workshop, January 1997, CERN-SL/97-06 (DI).

[9] H. Burkhardt, "Energy dependence of Beam-Beam Interactions at LEP”, contribution to this conference.

[10] G. Cavallari et al. "Beam Related Thermal Losses in the LEP SC RF System”, CERN LEP2 note 97-40 (21/4/97)

[11] S. Myers; "Synchro-Betatron Resonance Excitation in LEP", Proc. of the IEEE Particle Accelerator Conference held in Washington D.C., March 16-19, 1987, p. 1325.

[12] W. Herr, "Experience with Bunch Trains in LEP", contribution to this conference. 